\title{
Le temps des remises en question
}

\section{Le cas suisse}

Vocational education policy in transition in Switzerland

El tiempo de los cuestionamientos. El caso suizo

\section{Siegfried Hanhart}

\section{OpenEdition}

1 Journals

Édition électronique

URL : https://journals.openedition.org/ries/1711

DOI : 10.4000/ries. 1711

ISSN : 2261-4265

Éditeur

France Education international

\section{Édition imprimée}

Date de publication : 1 décembre 2003

Pagination : 109-116

ISBN : 978-2-8542-0558-9

ISSN : $1254-4590$

Référence électronique

Siegfried Hanhart, «Le temps des remises en question », Revue internationale d'éducation de Sèvres [En ligne], 34 | décembre 2003, mis en ligne le 23 novembre 2011, consulté le 09 juillet 2021. URL : http:// journals.openedition.org/ries/1711; DOI : https://doi.org/10.4000/ries.1711

(c) Tous droits réservés 


\section{Le temps des remises en question}

\section{Le cas suisse}

\section{Siegfried Hanhart}

Entre seize et vingt ans, une majorité de jeunes opte en Suisse pour une formation professionnelle. Pendant l'année scolaire 2001-2002, 310000 jeunes gens et jeunes filles de la classe d'âge susmentionnée se trouvaient en formation, dont près de $69 \%$ en formation professionnelle initiale (OFS, 2003). Il existe deux voies de formation professionnelle initiale : l'apprentissage en entreprise ou la formation en école professionnelle ou de métiers. Plus de huit jeunes sur dix optent pour l'apprentissage.

Longtemps l'adéquation entre la demande et l'offre de places d'apprentissage s'est opérée sans trop de difficultés. Cependant, le marché de l'apprentissage étant très sensible à la conjoncture, les autorités politiques ont dû intervenir à plusieurs reprises, depuis la décennie quatre-vingt-dix, afin d'inciter les entreprises à engager des apprentis. L'enjeu est à la fois économique et social : d'une part, il s'agit d'assurer à terme une relève de main-d'œuvre qualifiée; d'autre part, il faut éviter que des jeunes se retrouvent au chômage à l'issue de leur scolarité obligatoire, faute de trouver un emploi ou une possibilité de formation. Parallèlement aux mesures de stimulation du marché des apprentissages, les autorités politiques ont mis en œuvre de grands chantiers visant à accroître l'attrait de la formation professionnelle initiale et à développer la formation professionnelle supérieure. Ainsi, depuis quelques années l'offre de formation professionnelle dans le deuxième cycle de l'enseignement supérieur s'est enrichie d'une maturité professionnelle donnant accès à des Hautes écoles spécialisées (HES) au niveau de l'enseignement tertiaire ${ }^{1}$ : le nombre de jeunes obtenant une maturité professionnelle est en forte croissance, de même que les étudiants inscrits dans des HES. Enfin, une nouvelle loi fédérale sur la formation professionnelle entrera en vigueur en janvier 2004.

\section{LA PLACE DE LA FORMATION PROFESSIONNELLE INITIALE}

À l'issue de la scolarité obligatoire, les élèves peuvent poursuivre leur formation au deuxième cycle de l'enseignement secondaire dans l'enseignement général de type gymnasial ou acquérir une première formation professionnelle.

1. L'enseignement tertiaire désigne pour l'auteur l'enseignement supérieur. 


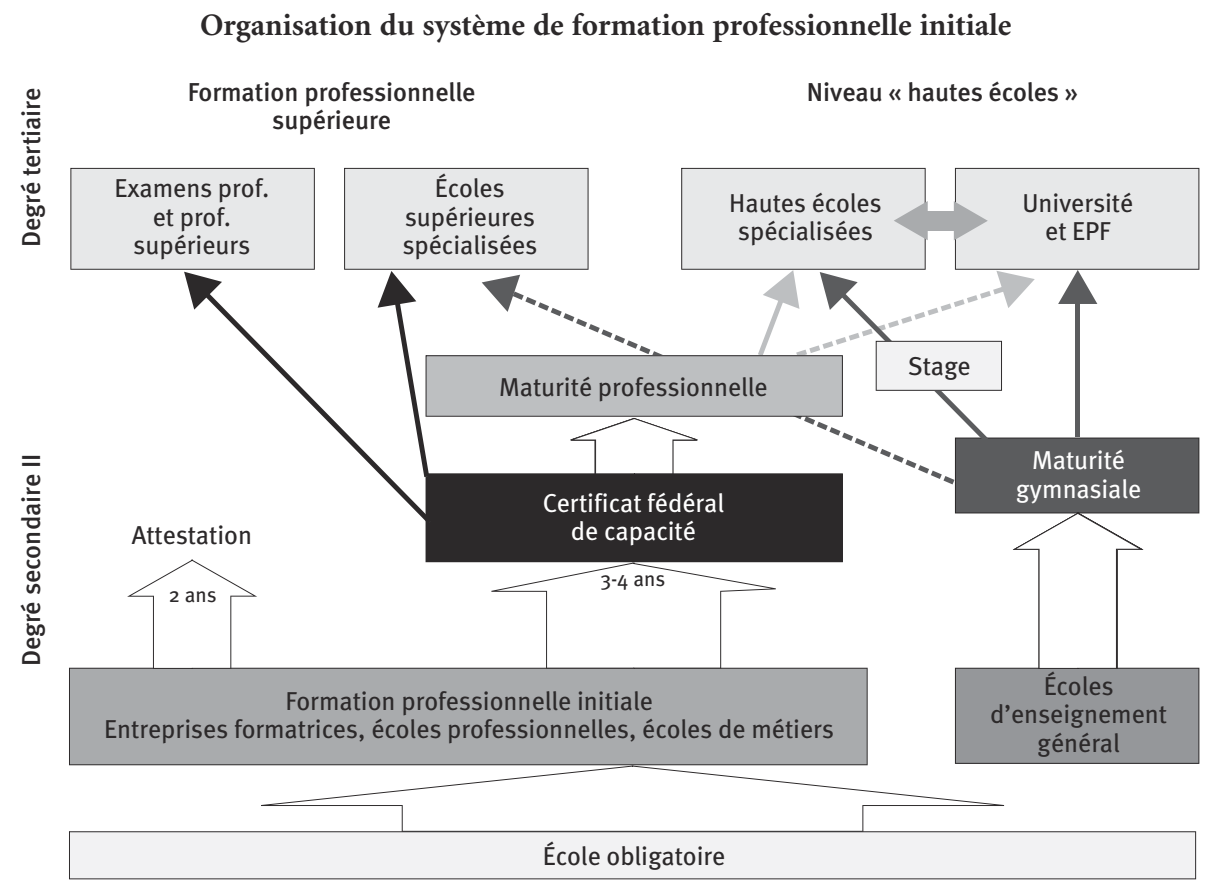

Source : OFFT, 2003 .

Le schéma ci-dessus permet de situer la formation professionnelle initiale dans le deuxième cycle du secondaire (sur le schéma désigné par «degré secondaire II») et indique les prolongements de la formation professionnelle initiale dans l'enseignement tertiaire. Les flèches désignent les transitions possibles du deuxième cycle du secondaire vers l'enseignement tertiaire et au sein de ce dernier.

En 2001, sept jeunes sur dix étaient engagés dans une des filières de la formation professionnelle du deuxième cycle $\mathrm{du}$ secondaire, les trois autres ayant opté pour un enseignement général. Relevons que la Suisse est le pays européen qui présente la proportion la plus élevée de jeunes entre seize et dix-neuf ans en formation professionnelle ${ }^{2}$. Ce phénomène n'est pas récent; dès la fin de la deuxième Guerre mondiale jusqu'au début des années quatrevingt-dix, la formation professionnelle a attiré de très nombreux jeunes et a fortement contribué à la qualification de la main-d'œuvre helvétique. Cependant, au cours de la dernière décennie, une proportion croissante de jeunes gens et de jeunes filles a choisi d'entrer dans des écoles d'enseignement général. 
Évolution comparative du nombre de jeunes en écoles de maturité et en formation professionnelle initiale (1990-2000)

\begin{tabular}{|l|r|r|r|}
\hline Nombre de jeunes & $\mathbf{1 9 9 0 / 9 1}$ & $\mathbf{1 9 9 5 / 9 6}$ & \multicolumn{1}{|c|}{$\mathbf{2 0 0 0 / 0 1}$} \\
\hline Dans des écoles préparant à la maturité & $\begin{array}{r}54311 \\
218780\end{array}$ & $\begin{array}{r}61833 \\
188510\end{array}$ & $\begin{array}{r}66888 \\
209157\end{array}$ \\
\hline En formation professionnelle initiale & \\
\hline
\end{tabular}

Source : Economiesuisse (2002).

Une comparaison de l'évolution des effectifs entre le nombre de jeunes préparant une maturité professionnelle en école professionnelle ou de métiers et le nombre de ceux engagés dans une formation professionnelle initiale en entreprise illustre ce mouvement.

Si nous comparons les effectifs des années 1990 et 1995, nous observons que le deuxième degré de l'enseignement secondaire a enregistré une diminution de 15600 individus consécutive à une évolution démographique négative. Il est intéressant de constater que les écoles de maturité ont vu entre les deux années de référence leurs effectifs augmenter de 7500 étudiants, tandis que la formation professionnelle perdait momentanément 30000 personnes! La période quinquennale suivante est caractérisée par une reprise de la croissance des effectifs dans le secondaire de deuxième degré; les écoles préparant à la maturité et les filières de formation professionnelle en bénéficieront.

Ce mode de formation concerne environ trois cents professions, dont la durée de formation est de trois ou quatre ans. Il s'agit d'un système dual ${ }^{3}$ : l'apprenti ${ }^{4}$ partage son temps entre l'école professionnelle (un jour et demi à deux jours par semaine) et l'entreprise (trois jours et demi à quatre jours). Il est lié à l'entreprise formatrice par un contrat spécifique et est considéré comme un salarié en formation, comme n'importe quel autre collaborateur. Il perçoit une rémunération variable selon le type d'apprentissage, l'année de formation et l'entreprise. En début de formation, les apprentis suivent des cours d'introduction dans des écoles professionnelles ou dans des centres de formation, voire dans des entreprises disposant d'ateliers d'apprentissage. À l'issue de la formation, l'apprenti qui réussit les examens obtient un certificat fédéral de capacité.

\section{L’ÉVOLUTION RÉCENTE DU MARCHÉ DE L'APPRENTISSAGE}

Si la formation de base en Suisse est caractérisée par une très nette prédominance de l'État (de l'enseignement primaire à l'enseignement universitaire), l'apprentissage constitue un véritable marché; la demande de places d'apprentissage

3. Il est aussi possible pour certaines professions (santé commerce, art) d'effectuer un apprentissage en étant à plein temps dans une école professionnelle.

4. Le terme d'apprentis désigne ici aussi bien des hommes que des femmes. 
est à la fois tributaire de la démographie et des préférences des jeunes entre formation professionnelle et enseignement général, et l'offre de la disponibilité des entreprises à accueillir des apprentis. A priori le fonctionnement du marché de l'apprentissage devrait présenter une analogie avec celui du travail.

Il présente, de fait, une grande sensibilité à l'évolution de la situation économique. Entre 1990 et 1994, la Suisse a connu une phase de récession suivie de trois années de très faible croissance, puis de 1997 à 2000, le PIB a augmenté entre 1,5 et $3 \%$ par an. Cependant, si les tendances sur les deux marchés sont analogues, les diminutions et les augmentations du nombre d'apprentis sont plus prononcées. La baisse plus marquée du nombre d'apprentis de 1990 à 1994 pourrait s'expliquer par le fait qu'en période de récession, les entreprises n'engagent plus de nouveaux collaborateurs, tendant d'abord à préserver les emplois des personnes déjà engagées. Le renouvellement des apprentis est ralenti au même titre que l'engagement de nouveaux collaborateurs. En revanche, le phénomène d'augmentation du nombre d'apprentis dès 1996 ne s'explique pas seulement par la reprise économique. Comme nous le montrerons par la suite, les pouvoirs publics ont mis en œuvre différentes mesures visant à encourager l'engagement d'apprentis, et ces mesures ont conduit à un accroissement substantiel de l'offre de places d'apprentissage.

Dans une période où les actionnaires et les directions des entreprises visent une rentabilité à court terme, l'on peut se demander si le coût de la formation ne représente pas un obstacle à l'accueil d'apprentis dans les entreprises. Soulignons que jusqu'en 1998, aucune étude exhaustive n'avait été publiée en Suisse sur les coûts et sur le financement de la formation des apprentis en entreprise! Les entreprises assument des dépenses de formation (coûts salariaux des apprentis, des formateurs internes et externes, matériel et équipement professionnels destinés à la formation, etc.) qui constituent les coûts bruts de formation. De ces coûts bruts, il convient de déduire la valeur de l'apport productif des apprentis pour obtenir les coûts nets. Dans une récente étude auprès de 2300 entreprises, Schweri \& alii (2003) montrent qu'en moyenne la formation des apprentis est économiquement rentable pour les entreprises formatrices. Ils estiment pour l'ensemble des entreprises formatrices l'apport productif des apprentis pour l'année 2000 à 5,2 milliards de francs suisses et les coûts bruts à 4,8 milliards, soit un bénéfice net de près de 400 millions. Cependant les auteurs de la recherche signalent que le rapport coûts/bénéfices varie considérablement selon la taille des entreprises, le secteur d'activité et selon les professions. Dans une autre recherche ${ }^{5}$, nous avons interrogé près de mille cinq cents entreprises formatrices pour connaître les raisons de leur engagement à former des apprentis; nous leur avions soumis douze arguments et nous ne mentionnons ci-après que les trois les plus souvent cités. Les entreprises ont d'abord mis l'accent sur le fait qu'en formant des

5. Hanhart, Schulz \& Bossio, 1998, pp. 112-124. 
apprentis elles génèrent un capital humain dont les qualifications correspondent à leurs besoins; les entreprises ont aussi exprimé la conviction que le système dual de formation professionnelle aboutit à des formations meilleures que celles qui sont dispensées dans des écoles professionnelles ou de métiers à plein temps. Enfin, elles ont souligné que la formation d'apprentis constituait aussi un moyen important de remédier à la pénurie de personnel qualifié. Il est à noter, cependant, que si l'apprentissage professionnel est populaire en Suisse, toutes les entreprises susceptibles d'accueillir des apprentis ne s'engagent pas ou plus dans la formation de jeunes. Nous avons également interrogé des entreprises non formatrices : celles qui n'ont jamais accueilli d'apprentis ont invoqué le manque de place, des activités trop spécialisées pour satisfaire aux exigences d'un apprentissage complet, ou encore l'inexistence d'apprentissage dans leur domaine d'activité; les entreprises qui ont renoncé à former des apprentis après en avoir accueilli, ont invoqué principalement le niveau scolaire insuffisant des candidats et le manque de temps pour encadrer les jeunes qui leur sont confiés.

Le tableau que nous venons d'esquisser pourrait donner à penser que l'apprentissage dual en Suisse ne rencontre pas de problèmes. Pourtant la très grande sensibilité à la conjoncture économique et la perte d'attraction de l'apprentissage par rapport aux études générales du deuxième cycle de l'enseignement secondaire ont obligé les autorités politiques fédérales à prendre des mesures visant à revitaliser cette voie de formation. Les interventions des pouvoirs publics ne s'inscrivent pas uniquement dans la perspective de réguler le marché de l'apprentissage, mais aussi dans le dessein de créer des conditions cadres favorables à la croissance économique. Par ailleurs, il n'est pas possible de passer sous silence la fonction sociale de l'apprentissage dans un pays où plus de $55 \%$ de la population active occupée a un niveau de qualification correspondant au deuxième degré de l'enseignement secondaire. De nombreux jeunes, désireux de quitter le système scolaire à quinze ou à seize ans, ont réussi leur transition vers le monde du travail grâce à l'apprentissage dual. Avec l'évolution des professions, les maîtres d'apprentissage tendent dans de nombreuses professions à privilégier le recrutement des jeunes issus des meilleures filières de l'enseignement secondaire de $1^{\text {er }}$ degré. Les adolescents dotés d'un moindre bagage scolaire éprouvent de plus en plus de peine à trouver un apprentissage et risquent à terme une marginalisation sociale. Il s'agit ici d'un autre enjeu important lié à l'avenir de l'apprentissage dual en Suisse.

\section{DES MESURES POUR REVITALISER L'APPRENTISSAGE}

Au $1^{\text {er }}$ janvier 2004 entrera en vigueur une nouvelle loi fédérale sur la formation professionnelle ${ }^{6}$. Cette refonte est destinée à faciliter l'adaptation de

6. Pour plus d'information sur cette nouvelle loi, le lecteur consultera http://www.bbt.admin.ch/dossiers/nbb/f/reform.htm 
la formation professionnelle à l'évolution des besoins de qualifications professionnelles. Nous nous limiterons ici à présenter les modifications qui touchent à la formation professionnelle initiale.

La nouvelle loi permet aux autorités politiques d'apporter plus rapidement des mesures correctives, par exemple sur le marché de l'apprentissage ${ }^{7}$. Au milieu des années quatre-vingt-dix, les autorités fédérales, face à la diminution du nombre de places d'apprentissage, avaient dû adopter d'urgence des arrêtés visant à encourager les entreprises à créer des opportunités de formation. Ces mesures à caractère exceptionnel et à durée limitée ont sans aucun doute connu un succès, si nous nous référons à l'augmentation du nombre d'apprentis dans la seconde partie de la dernière décennie.

La loi donne dorénavant la possibilité aux instances fédérales de réagir à la conjoncture avec plus de souplesse et de rapidité. Elle couvre aussi de nouveaux domaines de formation - la santé, les professions du social et des arts - qui précédemment faisaient l'objet de dispositions légales distinctes. Cette intégration débouchera sur de nouvelles offres de formation initiale dans le deuxième degré de l'enseignement secondaire.

La loi prévoit également des offres de formation initiale de deux ans destinées à des jeunes éprouvant des difficultés à suivre des formations de trois à quatre ans : dans le domaine des apprentissages, elle prévoit une diversification des modèles de formation visant à améliorer la rentabilité de l'apprentissage pour les entreprises (scolarité dégressive au long de l'apprentissage, possibilité de cours interentreprises). Jusqu'à ce jour, une entreprise formatrice devait s'engager à assurer la formation des jeunes pendant toute la durée de l'apprentissage. La nouvelle loi prévoit aussi la possibilité pour les entreprises formatrices de se regrouper en réseau. Sur le plan financier, la nouvelle législation offre l'opportunité aux autorités fédérales de créer un fonds en faveur de la formation professionnelle, dont le financement sera assuré par les branches d'activités et les entreprises qui ne participent pas à la couverture des frais de formation professionnelle.

Dans le courant des années quatre-vingt-dix, une nouvelle voie de formation professionnelle a été ouverte : la maturité professionnelle. Celle-ci est destinée à des jeunes désireux d'acquérir à la fois une pratique professionnelle et une culture générale permettant d'accéder aux hautes écoles spécialisées (HES) de l'enseignement tertiaire. La maturité professionnelle peut être préparée selon deux modalités : soit en la combinant avec l'apprentissage traditionnel en trois

7. Le conseiller fédéral en charge du département de l'économie a déjà eu recours à une mesure de ce genre en mars 2003. Face au risque qu'un nombre important de jeunes ne trouvent pas de place d'apprentissage à la rentrée scolaire 2003-2004, il a décidé de la mise sur pied d'une Task Force «Places d'apprentissage 2003». Cette dernière a pour mission de suivre l'évolution du marché des places d'apprentissage et de prendre des mesures le cas échéant. L'objectif premier de la Task Force est d'offrir à tous les jeunes sortant de l'école la possibilité de suivre une formation professionnelle en automne 2003. 
ou quatre ans, soit après l'obtention du certificat fédéral de capacité sanctionnant la réussite de l'apprentissage sous la forme d'une année supplémentaire en école professionnelle. L'introduction vise à rehausser l'attractivité de la formation professionnelle initiale du deuxième degré de l'enseignement secondaire auprès des jeunes, et à favoriser le développement de l'enseignement professionnel supérieur dans l'enseignement tertiaire. Cette voie de formation connaît un développement rapide, puisqu'en 1996480 titres étaient délivrés et $6478^{8}$ en 2000. Parallèlement à l'essor de la maturité, signalons le développement des hautes écoles spécialisées, dont la formation ne relève pas stricto sensu de la formation professionnelle initiale, mais qu'une bonne partie des usagers fréquente directement après avoir acquis une formation professionnelle dans le secondaire de deuxième degré. En 2002, les HES accueillaient près de 30000 étudiants; nous sommes certes encore loin des 105000 étudiants de l'enseignement universitaire, mais les prévisions pour 2010 envisagent plus de 40000 étudiants dans les HES! Le lecteur notera encore que les diplômés des HES connaissent très peu de chômage ${ }^{9}$, preuve que les formations dispensées répondent aux besoins des entreprises.

\section{UNE ÉVOLUTION LOURDE DE CONSÉQUENCES?}

Il aura fallu la crise des années quatre-vingt-dix, pour que les milieux politiques et économiques prennent conscience de l'urgence de réformes dans la formation professionnelle initiale. Dans un pays où un large consensus politique est nécessaire au changement dans les politiques sociales, il est frappant de constater la rapidité avec laquelle les décideurs politiques ont adopté des réformes aussi importantes que l'introduction de maturités professionnelles ou la création de hautes écoles spécialisées. Face à ces profondes mutations, il est nécessaire de s'interroger si l'on ne va pas progressivement vers une scolarisation et une académisation de la formation professionnelle; une telle évolution répondrait peut-être aux besoins d'une économie de services, mais elle risquerait aussi de rendre plus difficiles les transitions vers le marché du travail pour de nombreux jeunes peu à l'aise dans les systèmes scolaires. L'introduction de filières de formation de courte durée ne résoudra pas forcément les difficultés des populations à faible bagage scolaire. Cela nous amène à constater que la formation professionnelle initiale débute en réalité avant l'entrée en apprentissage ou en école professionnelle! Dès lors, il convient de prêter une attention particulière à la préparation à la transition entre la scolarité de base et la formation professionnelle initiale. Si l'on peut observer que la formation professionnelle initiale en Suisse semble s'adapter rapidement aux changements économiques,

8. Economiesuisse, 2002.

9. OFS, 2002. 
il nous semble que les préoccupations ces prochaines années devraient non seulement se traduire par la création ou la transformation de filières, mais aussi par le souci d'améliorer les possibilités pour tous les jeunes d'acquérir des qualifications scolaires qui leur permettront d'accéder à des formations professionnelles et au marché du travail ... et de s'y maintenir. Et pour ceux qui auraient «raté une première fois le train» l'opportunité de le rattraper ultérieurement. Nous entrons ici dans une perspective élargie de la formation professionnelle, celle de l'apprentissage à vie!

\section{BIBLIOGRAPHIE}

Economiesuisse (2002): Miroir statistique de la Suisse. Genève et Zurich.

HANHART S., SCHULZ H.-R. avec la coll. de BOSSIO S. (1998) : La formation des apprentis en Suisse. Coûts et financements. Lausanne : Delachaux et Niestlé.

OFFT (Office fédéral de la formation et de la technologie) (2003a): Système de la formation professionnelle en Suisse. Consulté le 22.9.2003 sur: http://www.bbt.admin.ch/berufsbi/system/f/

OFFT (Office fédéral de la formation et de la technologie) (2003b): Places d'apprentissage 2003. Tendances - Chiffres-Mesures. Berne.

OFS (Office fédéral de la statistique) (2003): Élèves et étudiants 2001/2002. Neuchâtel.

OFS (2002): De la haute école à la vie active. Premiers résultats de l'enquête 2001 auprès des nouveaux diplômés. Neuchâtel.

SCHWERI J., MÜHLEMANN S., PESCIO Y., WALTHER B., WOLTER S.C. \& ZÜRCHER L. (2003): Kosten und Nutzen der Lehrlingsausbildung aus der Sicht Schweizer Betriebe. Zürich/Chur : Verlag Rüegger. 